\title{
Dopamine receptor variants improve response to rasagiline in PD
}

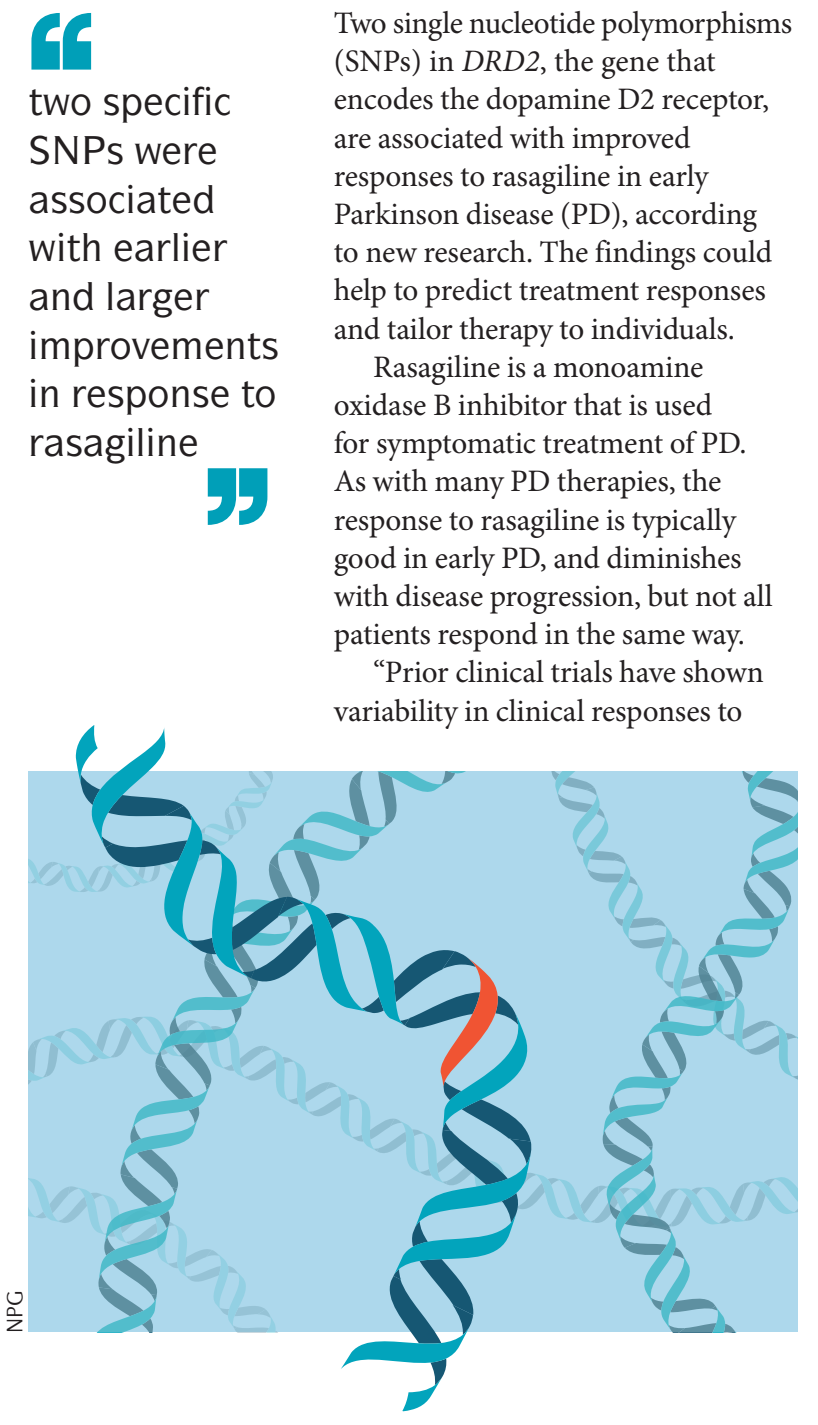

rasagiline," explains Mario Masellis, lead author of the new study. "We hypothesized that genetic variation may contribute to this inter-individual variability."

The researchers studied 692 participants of the ADAGIO trial, the largest published trial to look at clinical responses to rasagiline. The participants received rasagiline or a placebo during the first 36 weeks of the trial. Masellis and colleagues identified a set of 30 genes that influence either the pharmacokinetics and pharmacodynamics of rasagiline or relate to the pathogenesis of PD, and genotyped 209 genetic variants in DNA samples from the trial participants. They compared genetic variants with changes in Unified Parkinson Disease Rating Scale (UPDRS) scores at 12 weeks, 24 weeks and 36 weeks.

"This sort of large-scale analysis that combines genetic data from over 200 variants with clinical motor ratings has never been done before in relation to PD," says Masellis. For that reason, the analysis required collaboration with statistical geneticist Jo Knight, who added expertise in handling large genetic datasets.

Treatment with rasagiline improved PD symptoms to a greater extent than the placebo regardless of genotype, with a peak response at about 12 weeks. However, the analysis identified two specific SNPs — rs2283265 and rs1076560 — in $D R D 2$ that were associated with earlier and larger improvements in response to rasagiline, with a greater peak reduction in UPDRS scores at 12 weeks.

Masellis warns that these results should be considered preliminary, as they need to be replicated in independent cohorts. Nevertheless, he says that the genetic assocations are encouraging and the approach could be used in future to refine PD therapy.

"Pharmacogenetics and, more broadly, personalized medicine are exciting avenues to pursue in PD treatment, with the intention of eventually developing clinically useful point-of-care tests that can identify responders, nonresponders and those at risk of adverse effects," he says. However, he adds that more studies are needed before this option becomes a reality.

Ian Fyfe

ORIGINAL ARTICLE Masellis, M. et al. Dopamine D2 receptor gene variants and response to rasagiline in early Parkinson's disease: a pharmacogenetic study. Brain http://dx.doi. org/10.1093/brain/aww109 (2016) 